Pacific Journal of Mathematics

SOME REMARKS ON THE CALCULATION OF
STIEFEL-WHITNEY CLASSES AND A PAPER OF WU-Y

MCKENZIE Y. K. WANG 


\title{
SOME REMARKS ON THE CALCULATION OF STIEFEL-WHITNEY CLASSES AND A PAPER OF WU-YI HSIANG'S
}

\author{
MCKENZIE Y. K. WANG
}

In this paper we employ the techniques introduced by Wu-Yi Hsiang in [4] to perform Stiefel-Whitney class calculations for the possibilities of connected principal isotropy type listed in Theorems 1-3 of [4]. We show that some of the possibilities listed there do not occur if we assume in addition that sufficiently many Stiefel-Whitney classes of the $G$-manifold vanish. We therefore obtain a slightly shorter list of possibilities of connected principal isotropy type for compact connected Lie group actions on parallelizable manifolds. Stiefel manifolds which are not spheres, for example, fall under this category. We also give an example of how our results may be used to study actions on Stiefel manifolds. As this paper is actually a supplement to [4], we refer the reader to it for notation and general philosophy.

1. Preliminaries on Stiefel-Whitney classes and 2-weights. In this section we set the stage for our calculations, which will be described in the next section. Basically, the statements here are parallel to those in $\S \S 1$ and 2 of [4].

Definition. Let $\psi: H \rightarrow G$ be a homomorphism of compact Lie groups. Suppose $P$ and $Q$ are, respectively, maximal 2-tori of $H$ and $G$, and $\psi(P) \subseteq Q$. Let $\left\{y_{i}\right\}$ be a basis for $\operatorname{Hom}(Q, Z / 2) \approx H^{1}(B Q, Z / 2)$ and $\psi^{*}: H^{1}(B Q, Z / 2) \rightarrow H^{1}(B P, Z / 2)$. Then $\left\{x_{i}=\psi^{*} y_{i}\right\}$ are called the 2weights of $\psi$ relative to $(P, Q)$. In particular, if $\psi=\operatorname{Ad} H, G=O(\mathfrak{h})$, then the 2-weights are called the 2-roots of $H$ relative to the maximal 2-tori $(P, Q)$.

We list below the non-zero 2-roots of some classical groups.

\begin{tabular}{|l|c|c|c|}
\hline$G$ & $Q$ & 2-roots & multiplicity \\
\hline $\mathrm{U}(n)$ & $\operatorname{diag}\left(\varepsilon_{1}, \ldots, \varepsilon_{n}\right), \varepsilon_{l}= \pm 1$ & $y_{i}-y_{J}, i<j$ & 2 \\
$\mathrm{SU}(n)$ & $\operatorname{diag}\left(\varepsilon_{1}, \ldots, \varepsilon_{n}\right), \varepsilon_{l}= \pm 1$ & & 2 \\
& $\sum_{1}^{n} \varepsilon_{l}=0$ & & \\
& $\operatorname{diag}\left(\varepsilon_{1}, \ldots, \varepsilon_{n}\right), \varepsilon_{l}= \pm 1$ & $\prime \prime$ & 1 \\
$\mathrm{O}(n)$ & $\operatorname{diag}\left(\varepsilon_{1}, \ldots, \varepsilon_{n}\right), \varepsilon_{l}= \pm 1$ & $\prime \prime$ & 1 \\
$\mathrm{SO}(n)$ & $\sum^{n} \varepsilon_{l}=0$ & & \\
& 1 & & 4 \\
$\mathrm{Sp}(n)$ & $\operatorname{diag}\left(\varepsilon_{1}, \ldots, \varepsilon_{n}\right), \varepsilon_{l}= \pm 1$ & $\prime \prime$ & \\
\hline
\end{tabular}


We shall also need to know the maximal 2-tori in the exceptional Lie groups $G_{2}$ and $F_{4}$. We refer the reader to the papers $[1,2]$ for details. However, to summarize, let us mention that if the Cayley numbers are given by $R+R e_{1}+R e_{2}+R e_{3}+\cdots+R e_{7}$, then $G_{2}$ is the group Aut(Cay). Suppose $S_{l}$ : Cay $\rightarrow$ Cay are given by $S_{t}\left(e_{t+1}\right)=e_{i+1}, S_{i}\left(e_{t+5}\right)$ $=e_{l+5}, S_{l}\left(e_{i+6}\right)=e_{l+6}$ and $S_{i}\left(e_{j}\right)=-e_{j}$ if $j \neq i+1, i+5, i+6$. Then $Q=\left\{1, S_{1}, \ldots, S_{7}\right\}$ are the automorphisms of the Cayley numbers which form a maximal 2-torus of $G_{2}$. We let $x_{i}$ in $\operatorname{Hom}(Q, Z / 2)$ be given by $x_{i}\left(S_{j}\right)=\delta_{i j}$. The 2-roots of $G_{2}$ with respect to $Q$ are $x_{i}+x_{\jmath}, i<j$, with multiplicity $2, x_{1}+x_{2}+x_{3}$ with multiplicity 2 , together with $x_{i}$ with multiplicity 2 .

In the case of $F_{4}$, note that $\operatorname{Spin}(9) \subseteq F_{4}$ and every maximal 2-torus in $F_{4}$ is conjugate to one in $\operatorname{Spin}(9)$. Consequently, we may take the maximal 2-tori in $\operatorname{Spin}(9)$ to be maximal 2-tori in $F_{4}$. Now $G_{2} \subseteq \operatorname{Spin}(7) \subseteq \operatorname{Spin}(8)$ $\subseteq \operatorname{Spin}(9)$, and center $(\operatorname{Spin}(7)) \simeq Z / 2$, center $(\operatorname{Spin}(8)) \simeq(Z / 2)^{2}$. Therefore, we may parametrize a maximal 2-torus in $\operatorname{Spin}(9)$ by $x_{1}, x_{2}, x_{3}, y, z$, where the $x_{i}$ 's come from $G_{2}, y$ from center(Spin(7)) and $z$ from

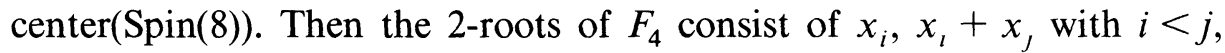
$x_{1}+x_{2}+x_{3}$, all with multiplicity 4, together with $y, y+x_{i}, y+x_{t}+x_{j}$, $y+x_{1}+x_{2}+x_{3}, z, z+x_{l}, z+x_{i}+x_{j}, z+x_{1}+x_{2}+x_{3}, y+z, y+z$ $+x_{l}, y+z+x_{l}+y_{j}, y+z+x_{1}+x_{2}+x_{3}$ with multiplicity 1 and $i<j$.

Proposition. Let $\xi$ be a principal $H$-bundle, where $H$ is compact Lie. Let $Q$ be a maximal 2-torus of $H$ and $\Phi: H \rightarrow \mathrm{SO}(n)$ (respectively $\mathrm{O}(n)$, $\mathrm{SU}(n), \mathrm{U}(n), \mathrm{Sp}(n))$ a representation of $H$. Assume $\Phi(Q) \subseteq Q^{\prime}$, the standard maximal 2-torus of $\mathrm{SO}(n)$ (respectively $\mathrm{O}(n), \mathrm{SU}(n), \mathrm{U}(n)$, $\operatorname{Sp}(n))$. Let $\pi: E(\xi) / Q \rightarrow B(\xi)$ and $\left\{w_{i}\right\}$ be the 2-weights of $\Phi$ with respect to $\left(Q, Q^{\prime}\right)$ counted with multiplicity. If $\eta$ is the extension of $\xi$ by the representation $\Phi$, and $i: E(\xi) / Q \rightarrow B Q$ is the classifying map of the principal $Q$-bundle $Q \rightarrow E(\xi) \rightarrow E(\xi) / Q$, then

$$
i^{*} \prod_{w_{\imath} \in \Omega_{2}(\Phi)}\left(1+w_{l}^{d}\right)=\pi^{*}\left(w_{*} \eta\right),
$$

where $d=1,2,4$, and $w_{*} \eta$ denotes the total Stiefel-Whitney class, the total Chern class reduced mod 2 , or the total quaternion Pontrjagin class reduced mod 2. $\Omega_{2}(\Phi)$ denotes the system of 2-weights of the representation $\Phi$.

Proof. This is basically Theorem 11.3 in [1]. We have a commutative diagram 


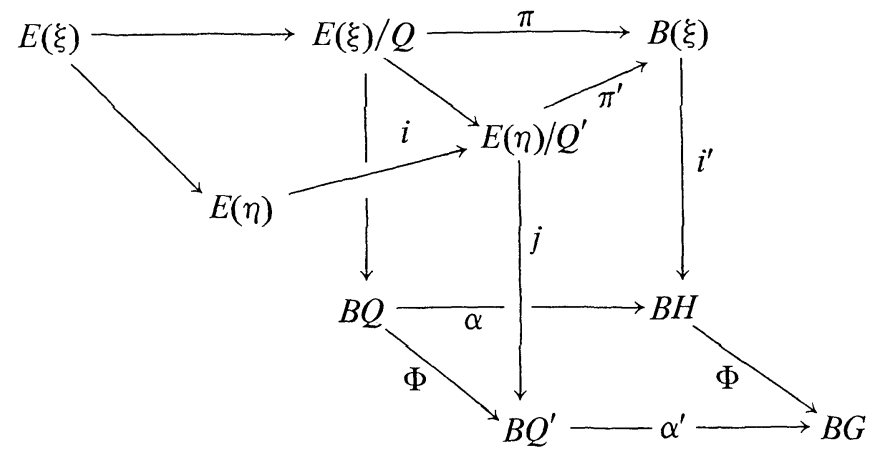

We consider first the cases of $\mathrm{SO}(n)$ and $\mathrm{O}(n)$. For this recall that $H^{*}(\mathrm{BO}(n) ; Z / 2) \rightarrow H^{*}\left(B Q^{\prime} ; \quad Z / 2\right) \simeq Z / 2\left[y_{1}, \ldots, y_{n}\right]$ identifies $H^{*}(\mathrm{BO}(n) ; Z / 2)$ with $Z / 2\left[w_{1}, \ldots, w_{n}\right] \subseteq Z / 2\left[y_{1}, \ldots, y_{n}\right]$. The $w_{l}$ 's are elementary symmetric polynomials in the $y_{l}$ 's which form a basis for $H^{1}\left(B Q^{\prime} ; Z / 2\right)$. Now

$$
\pi^{*}\left(w_{*} \eta\right)=\pi^{*} i^{\prime *} \Phi^{*} w_{*}=i^{*} \alpha^{*} \Phi^{*} w_{*}=i^{*} \Phi^{*} \alpha^{*} w_{*},
$$

where $w_{*}$ denotes the total universal Stiefel-Whitney class. But $\alpha^{\prime *} w_{*}=$ $\prod_{l=1}^{n}\left(1+y_{l}\right)$, so

$$
\begin{aligned}
\pi^{*}\left(w_{*} \eta\right) & =i^{*} \Phi^{*}\left(\prod_{i=1}^{n}\left(1+y_{l}\right)\right)=i^{*} \prod_{1}^{n}\left(1+\Phi^{*} y_{l}\right) \\
& =i^{*} \prod_{w_{i} \in \Omega_{2}(\Phi)}\left(1+w_{i}\right) .
\end{aligned}
$$

For the case of $\mathrm{SO}(n)$, the only difference is that $w_{1}=0$.

Next we consider the parallel cases of $\mathrm{SU}(n), \mathrm{U}(n)$ and $\mathrm{Sp}(n)$. As an example, we discuss the case of $U(n)$ in detail. The only observation we have to make is that

$$
H^{*}(\mathrm{BU}(n) ; Z / 2)=Z / 2\left[c_{1}, \ldots, c_{n}\right] \subseteq Z / 2\left[y_{1}, \ldots, y_{n}\right] \simeq H^{*}\left(B Q^{\prime} ; Z / 2\right),
$$

where $c_{l}$ is the $i$ th elementary symmetric polynomial in the $y_{i}^{2}$ 's. Therefore, if $c_{*}$ is the universal total Chern class reduced mod 2, $c_{*}=\Pi\left(1+y_{i}^{2}\right)$. This accounts for the power of 2 in the proposition. For $\mathrm{SU}(n), c_{1}=0$. The case of $\operatorname{Sp}(n)$ is similar.

Let $H$ be a compact connected Lie group and $\Psi: H \rightarrow \mathrm{SO}(m)=G$ (respectively $\mathrm{SU}(m), \mathrm{Sp}(m)$ ) almost faithful representations of $H$. We should think of $\Psi(H)$ as the connected component of some isotropy group of a classical group action on a compact manifold $M$ which has some vanishing Stiefel-Whitney classes. Let $x \in M$ and $\Psi(H)=G_{x}^{0}$. 
Then we have $G / \Psi(H) \stackrel{p}{\rightarrow} G / G_{x} \stackrel{h}{\rightarrow} G(x) \stackrel{j}{\rightarrow} M$. Employing reduction 2 in $\S 1$ of [4], we have

$$
\tau(G / \Psi(H))=p ! \tau\left(G / G_{x}\right)=p ! h ! \tau(G(x))
$$

in $\tilde{\mathrm{K}} \mathrm{O}(G / \Psi(H))$. It is well known that $\tau(G / \Psi(H))$ is the extension of the principal $\Psi(H)$ bundle $\Psi(H) \rightarrow G \rightarrow G / \Psi(H)$ by the isotropy representation. If $x$ is on a principal orbit, then $\nu(G(x))$, the normal bundle to the orbit through $x$, is trivial. Since Stiefel-Whitney classes obey a product formula and are stable characteristic classes, vanishing conditions on the Stiefel-Whitney classes of $M$ pull back to vanishing conditions on those of $\tau(G / \Psi(H))$.

Let us denote the principal $\Psi(H)$ bundle above by $\alpha(\Psi, H)$. Then

$$
\tau(G / \Psi(H))=\alpha(\Psi, H)(\operatorname{Ad} G \mid \Psi(H)-\operatorname{Ad} \Psi(H)),
$$

so the fundamental observation of Hsiang (reduction 4 in $\$ 1$ of [4]) allows us to transfer the vanishing conditions on $w_{l}(G / \Psi(H))$ to $w_{l}(\alpha(\Psi, H)(\operatorname{Ad} \Psi(H)))$. In Proposition 1, we can set $\xi=\alpha(\Psi, H), \Phi=$ $\operatorname{Ad} \Psi(H)$, and $\left\{\alpha_{l}\right\}=$ the 2-roots of $\operatorname{Ad} \Psi(H)$ counted according to multiplicity. Then provided that $\Phi(\Psi(Q)) \subseteq Q^{\prime}$, we have

$$
\pi^{*}\left(w_{*} \eta\right)=i^{*}\left(\prod_{\alpha_{\imath} \in \Delta_{2}(\Phi)}\left(1+\alpha_{i}\right)\right) .
$$

As in [4] we must determine Ker $i^{*}$. The arguments in [4] go through if we replace $T$ by $\Psi(Q)$ and if we use $Z / 2$ coefficients. We must, however, justify the existence of the Serre spectral sequence for the fibration $\pi_{G}: E_{G} \times_{\Psi(Q)} G \rightarrow B \Psi Q$, since the base is not simply-connected. However, we simply have to note that we have here a principal $G$-bundle with $G$ connected, and it is well-known that this is a case where we have simple coefficients.

To compute $\operatorname{Ker} i^{*}$, let us consider $G=\operatorname{SU}(m)$ or $\operatorname{Sp}(m)$ first. These groups have no torsion. Let $c_{*}$ be the total universal Chern class reduced $\bmod 2$ in $H^{*}(\mathrm{BSU}(m) ; Z / 2)$, and $q_{*}$ the total universal quaternionic Pontrjagin class reduced $\bmod 2$ in $H^{*}(\mathrm{BSp}(m) ; Z / 2)$. Then the argument in [4] shows that $\operatorname{Ker} i^{*}=\operatorname{Ker} \pi_{G}^{*}=$ the ideal in $H^{*}(B \Psi Q ; Z / 2)$ generated by the images of all differentials of the Serre spectral sequence of $\pi_{G}$. If $\lambda: B \Psi Q \rightarrow B G$ is the classifying map of the principal $G$-bundle $\pi_{G}$, Ker $i^{*}=$ the ideal in $H^{*}(B \Psi Q ; Z / 2)$ generated by $\left\{\lambda^{*} c_{l}\right\}$ or, in the case of $\operatorname{Sp}(m)$, by $\left\{\lambda^{*} q_{i}\right\}$. We apply Proposition 1 again with $\xi=\left[\Psi Q \rightarrow E_{G} \rightarrow\right.$ $B \Psi Q], H=\Psi(Q), \Phi=$ the inclusion of $\Psi(Q)$ into $G, \eta=\pi_{G}, i=\mathrm{id}$ to obtain $\lambda^{*} c_{*}=\Pi_{w_{i} \in \Omega_{2}(\Phi)}\left(1+w_{i}^{2}\right)$ or $\lambda^{*} q_{*}=\prod_{w_{i} \in \Omega_{2}(\Phi)}\left(1+w_{i}^{4}\right)$. So Ker $i^{*}$ 
is the ideal $\left((W \Phi)_{2}^{2}, \ldots,(W \Phi)_{m}^{2}\right)$ for $G=\mathrm{SU}(m)$ and $\left((W \Phi)_{1}^{4}, \ldots,(W \Phi)_{m}^{4}\right)$ for $G=\operatorname{Sp}(m)$, where $(W \Phi)_{j}$ is the $j$ th elementary symmetric polynomial in the $w_{i}$ 's.

$$
w_{i} \in \Omega_{2}(\Phi) .
$$

If $G=\mathrm{SO}(m)$, then $G$ has 2-torsion. However, the generators of $H^{*}(\mathrm{SO}(m) ; Z / 2) \simeq \Delta\left(x_{1}, \ldots, x_{m-1}\right)$ are universally transgressive. So the problems of transgression encountered in Pontrjagin classes actually do not occur here. Thus exactly the same argument as in the previous cases yields $\operatorname{Ker} i^{*}=$ the ideal $\left((W \Phi)_{2}, \ldots,(W \Phi)_{m}\right)$.

REMARK. We emphasize here that the weights $w_{l}$ in the above discussion lie in $H^{1}(B \Psi Q ; Z / 2)$ and the equation

$$
\pi^{*}\left(W_{*} \eta\right)=i^{*} \prod_{\alpha_{i} \in \Delta_{2}(\Phi)}\left(1+\alpha_{i}^{d}\right)
$$

belongs to $H^{*}\left(E_{\alpha(\Psi, H)} / \Psi Q ; Z / 2\right)$. Order two elements in $Q$ may very well go to 0 under $\Psi$. This will be a point for us to be careful about in our calculations. However, it turns out that there are always enough points in our 2-tori so that we need not worry about elements going to 0 .

2. Symmetric products of 2-roots of simple Lie groups. In this section we shall compute certain symmetric products of the 2-roots of some of the simple Lie groups. If $K$ is a compact connected Lie group, we let $W K_{i}$ denote the $i$ th symmetric product of the 2-roots of $K, S K_{l}$ denote the $i$ th symmetric sum of the 2-roots of $K$. If $y_{1}, \ldots, y_{n}$ are variables, then $\sigma_{i}$ will denote the $i$ th symmetric polynomial in the $y_{j}$ 's. $s_{i}$ will denote the $i$ th symmetric sum in the $y_{j}$ 's.

We begin with the observation that $W \mathrm{SO}(n)_{*}=\Pi_{l<j}\left(1+y_{l}+y_{j}\right)$, $W \mathrm{SU}(n)_{*}=\Pi_{i<j}\left(1+y_{l}+y_{j}\right)^{2}$, and $W \mathrm{Sp}(n)_{*}=\Pi_{l<j}\left(1+y_{i}+y_{j}\right)^{4}$. Formally we must have $W \mathrm{SU}(n)_{2 i}=W \mathrm{SO}(n)_{l}^{2}$. For the case of $\operatorname{Sp}(n)$, if we use the sub-2-torus of the standard 2-torus, consisting of $\operatorname{diag}\left(\varepsilon_{1}, \ldots, \varepsilon_{n}\right)$, $\varepsilon_{l}= \pm 1, \sum \varepsilon_{i}=0$, then we have $\tilde{W} \mathrm{Sp}(n)_{4_{l}}=W \mathrm{SO}(n)_{i}^{4}$. We note the proof of Proposition 1 does not use the maximality of $Q$. Neither does the discussion of the vanishing conditions and Ker $i^{*}$. All this means that while symmetric products of 2-roots of $\mathrm{SU}(n)$ and $\mathrm{Sp}(n)$ may be hard to compute directly, they can be obtained from the results of $\operatorname{SO}(n)$. We therefore begin by computing $W \mathrm{SO}(n)_{i}$.

Let $k(n)$ be $2 n+1$ if we are considering $\operatorname{SO}(2 n+1)=B_{n}$, and $k(n)=2 n$ if we are considering $\operatorname{SO}(2 n)=D_{n}$. The basic technique of calculation is the use of Newton's formulas, followed by reduction mod 2 . 
Proposition. Let $W_{i}$ denote the ith symmetric product of the 2-roots of the Lie group H. Then we have:

(a) $H=\mathrm{SU}(n)=A_{n-1}$,

$W H_{\text {odd }}=0$,

$W H_{2}=0$,

$W H_{4}=n \sigma_{2}^{2}$,

$W H_{6}=n \sigma_{3}^{2}$,

$W H_{8}= \begin{cases}([n / 2]+1) \sigma_{2}^{4}+\sigma_{4}^{2} & \text { if } n \text { is odd }, \\ ([n / 2]+1) \sigma_{2}^{4} & \text { if } n \text { is even }\end{cases}$

$W H_{10}= \begin{cases}\sigma_{5} & \text { if } n \text { is odd, } \\ 0 & \text { if } n \text { is even, }\end{cases}$

$W H_{12}= \begin{cases}\sigma_{6}^{2}+([n / 2]+1) \sigma_{2}^{6}+([n / 2]+1) \sigma_{3}^{4} & \text { if } n \text { is odd }, \\ ([n / 2]+1) \sigma_{3}^{4} & \text { if } n \text { is even } ;\end{cases}$

(b) $H=\operatorname{SO}(2 n+1)$,

$W H_{1}=0, \quad W H_{2}=\sigma_{2}, W H_{3}=\sigma_{3}$,

$W H_{4}=\sigma_{4}+(n+1) \sigma_{2}^{2}$,

$W H_{5}=\sigma_{5}$,

$W H_{6}=\sigma_{6}+(n+1) \sigma_{2}^{3}+(n+1) \sigma_{3}^{2}$;

(c) $H=\operatorname{Sp}(n)=C_{n}$,

$W H_{\text {odd }}=0=W H_{2}$,

$W H_{4}=(n+1) \sigma_{1}^{4}$,

$\tilde{W} H_{4}=0$,

$\tilde{W} H_{8}=n \sigma_{2}^{4}$,

$\tilde{W} H_{12}=n \sigma_{3}^{4}$,

$\tilde{W} H_{16}= \begin{cases}\sigma_{4}^{4}+([n / 2]+1) \sigma_{2}^{8} & \text { if } n \text { is odd, } \\ ([n / 2]+1) \sigma_{2}^{8} & \text { if } n \text { is even; }\end{cases}$

(d) $H=\mathrm{SO}(2 n)$,

$$
\begin{aligned}
& W H_{1}=W H_{2}=W H_{3}=0, \\
& W H_{4}=(n+1) \sigma_{2}^{2}, \\
& W H_{5}=0, \\
& W H_{6}=(n+1) \sigma_{3}^{2}, \\
& W H_{8}=n \sigma_{4}^{2}+\sigma_{2}^{4}+\left(\left(\frac{11}{6}\right) n^{2}+\left(\frac{2}{3}\right) n^{4}+\left(\frac{5}{2}\right) n\right) \sigma_{2}^{4}+\sigma_{3} \sigma_{5},
\end{aligned}
$$


(e) $\left(W G_{2}\right)_{*}=1+\left(\sigma_{1}^{8}+\sigma_{1}^{2} \sigma_{3}^{2}+\sigma_{2}^{4}\right)$

$$
\begin{aligned}
& +\left(\sigma_{1}^{6} \sigma_{3}^{2}+\sigma_{3}^{4}+\sigma_{1}^{2} \sigma_{2}^{2} \sigma_{3}^{2}+\sigma_{1}^{4} \sigma_{2}^{4}\right) \\
& +\left(\sigma_{3}^{4} \sigma_{1}^{2}+\sigma_{1}^{4} \sigma_{2}^{2} \sigma_{3}^{2}\right)
\end{aligned}
$$

(f) Let

$$
\begin{aligned}
& A=\sigma_{1}^{4}+\sigma_{1} \sigma_{3}+\sigma_{2}^{2}, \quad B=\sigma_{1}^{3} \sigma_{3}+\sigma_{3}^{2}+\sigma_{1} \sigma_{2} \sigma_{3}+\sigma_{1}^{2} \sigma_{2}^{2}, \\
& C=\sigma_{3}^{2} \sigma_{1}+\sigma_{1}^{2} \sigma_{2} \sigma_{3},
\end{aligned}
$$

and

$$
\begin{aligned}
W(u)= & 1+u+u^{2}+u^{3}+\left(u^{4}+A\right)+\left(u^{5}+A u\right) \\
& +\left(u^{6}+A u^{2}+B\right)+\left(u^{7}+A u^{3}+C\right) .
\end{aligned}
$$

Then $\left(W F_{4}\right)_{*}=W(0)^{4} W(y) W(z) W(y+z)$. In particular, $\left(W F_{4}\right)_{*} \neq 1$ since for $y=1, z=x_{1}=x_{2}=x_{3}=0,\left(W F_{4}\right)_{*}=0=1+\left(W F_{4}\right)_{1}+\cdots$.

Proof. Among (a)-(d), the essential calculations occur with the $\mathrm{SO}(n)$ 's by a previous remark. Let $k(n)$ be $2 n+1$ or $2 n$ as described. It is clear that $W H_{1}=0$.

We have $W_{2}=\left(\frac{-1}{2}\right) S H_{2}=\left(\frac{-1}{2}\right) k(n) s_{2}=k(n) \sigma_{2}=\sigma_{2}$ for $k(n)=$ $2 n+1$ and 0 for $k(n)=2 n$. $S H_{3}=(k(n)-4) s_{3}=(k(n)-4) 3 \sigma_{3}$. So $W H_{3}=(k(n)-4) \sigma_{3}=k(n) \sigma_{3}$. Similarly, $S H_{4}=k(n) s_{4}+\left(\frac{1}{2}\right)\left(\begin{array}{l}4 \\ 2\end{array}\right) s_{2}^{2}=$ $k(n)\left(-4 \sigma_{4}+2 \sigma_{2}^{2}\right)+3\left(4 \sigma_{2}^{2}\right)=-4 k(n) \sigma_{4}+2 k(n) \sigma_{2}^{2}+12 \sigma_{2}^{2} . W H_{4}=$ $-\left(\frac{1}{4}\right)\left(-4 k(n) \sigma_{4}+2 k(n) \sigma_{2}^{2}+12 \sigma_{2}^{2}\right)+\left(\frac{1}{8}\right) k(n)^{2} 4 \sigma_{2}^{2} \equiv \sigma_{4}+(n+1) \sigma_{2}^{2}$ if $k(n)=2 n+1$ and $(n+1) \sigma_{2}^{2}$ if $k(n)=2 n$. Other calculations are similar, though the demand for perseverance increases.

We obtain the formulas for (a) by either direct computation or by the fact that $W \mathrm{SU}(n)_{2 i}=W \mathrm{SO}(n)_{i}^{2}$. The formulas in (c), (e), (f) are obtained by direct calculation or by the relationship $\tilde{W} \operatorname{SP}(n)_{4 l}=W \mathrm{SO}(n)_{i}^{4}$, where $\tilde{W}$ are the symmetric products calculated using the corank 1 sub-2-torus of the maximal 2-torus chosen.

3. Connected principal isotropy types and Stiefel-Whitney classes. Let $G$ denote $\mathrm{SO}(m), \mathrm{SU}(m)$, or $\mathrm{Sp}(m)$ and let $G$ act smoothly on a manifold $M$ whose first three Pontrjagin classes and all Stiefel-Whitney classes vanish. As can be seen, we do not really need the full force of the latter vanishing condition. For applications, we have in mind all stably parallelizable manifolds whose first three Pontrjagin classes vanish. For example, Stiefel manifolds and sufficiently high-dimensional homotopy spheres have this property. 
At this point we refer the reader to Theorems 1-3 in [4]. Basically, we show that certain cases in these theorems cannot occur if Stiefel-Whitney classes vanish. As described at the end of $\S 1$, if $\Psi: H \rightarrow G$ is an almost faithful representation of $H$ and $\Phi=\operatorname{Ad}(\Psi(H))$ takes $\Psi(Q)$ to a subtorus of $Q^{\prime}$, then we have the following vanishing condition:

$$
(W H)_{1}=0 \quad \bmod \left((W \Phi)_{1}^{d}, \ldots,(W \Phi)_{[i / d]}^{d}\right), \quad d=1,2,4,
$$

where $W H_{i}$ is the $i$ th symmetric product of the 2-roots of $\Psi(H)$ and $(W \Phi)_{l}=$ the $i$ th symmetric product of the 2-weights of the representation $\Phi: \Psi(H) \subseteq G$. We recall the remark in $\S 2$ that the 2-torus $\Psi(Q)$ need not be maximal in $\Psi(H)$, so that the above vanishing condition holds for any sub-2-torus $Q$ of $H$.

Proposition 1. Let $\mathrm{SU}(n)$ act smoothly on a manifold $M$ such that the first three Pontrjagin classes and all Stiefel-Whitney classes vanish. Let $\Psi$ : $H \rightarrow \mathrm{SU}(m)$ be an almost faithful complex representation of a compact connected Lie group $H$. Then for the following pairs of $(H, \Psi), \Psi(H)$ cannot be the connected principal isotropy type of the $\mathrm{SU}(m)$ action:

1. $H$ is semisimple, connected, compact, $\Psi=$ Ad.

2. $H=\mathrm{SU}(l) \times H_{1}$, ldivides $30, \Psi=\mu_{l} \otimes \mu_{l}+\operatorname{Ad} H_{1}$.

3. $H=\mathrm{SU}(l), \Psi=2 \mu_{l}$, and $l$ is odd.

4. $H=\mathrm{SO}(l), \Psi=\rho_{l}, l$ odd .

5. $H=\operatorname{Sp}(l), \Psi=\nu_{l}$, l even.

6. $H=G_{2}, \Psi=2 \Lambda_{2}, \Lambda_{2}$ is the 7-dimensional representation of $G_{2}$.

7. $H=G_{2} \times G_{2}, \Psi=2\left(\Lambda_{2}+\Lambda_{2}^{\prime}\right)$.

8. $H=\mathrm{SU}(3) \times \mathrm{SU}(3), \Psi=2\left(\mu_{3}+\mu_{3}^{\prime}\right), 2\left(\bar{\mu}_{3}+\bar{\mu}_{3}^{\prime}\right),\left(\mu_{3}+\mu_{3}^{\prime}\right)+$ $\left(\bar{\mu}_{3}+\bar{\mu}_{3}^{\prime}\right)$.

9. $H=\mathrm{SU}(3)$ or $\mathrm{SU}(5), \Psi=\mu_{l}+\bar{\mu}_{l}$.

10. $H=\mathrm{SU}(3), \Psi=k \mu_{3}+l \bar{\mu}_{3}, k+l=6$.

Proof. 1. Since $\Psi$ is allowed to be almost faithful, we may assume $H=H_{1} \times \cdots \times H_{k}$, where $H_{i}$ are simple normal factors of $H$. By reduction 2 in [4], one easily sees that we may assume $H$ itself is simple.

(a) $H=\mathrm{SU}(l)$.

If $l$ is even, then $Z(\mathrm{SU}(l))$ contains only one element of order 2 of the standard maximal 2-torus. Therefore we may performs the 2-weights calculation in $\mathrm{SU}(l)$. The vanishing condition is

$$
W H_{i} \equiv 0 \bmod \left(W H_{2}^{2}, \ldots, W H_{l}^{2}\right) .
$$


Now $W H_{8}=\left(\left(\frac{1}{2}\right) l+1\right) \sigma_{2}^{4}$ while $W H_{2}^{2}=0, W H_{4}^{2}=0$. This forces $\left(\frac{1}{2}\right) l$ to be odd. On the other hand,

$$
\begin{gathered}
W H_{16}=W \mathrm{SO}(l)_{8}^{2}=\sigma_{4}^{4}+C \sigma_{2}^{8}+\sigma_{3} \sigma_{5}, \\
W H_{2}^{2}=0=W H_{4}^{2}=W H_{6}^{2}=W H_{8}^{2}
\end{gathered}
$$

because $\left(\frac{1}{2}\right) l$ is odd. Therefore, $l$ must be odd. In this case $Z(\operatorname{SU}(l))$ contains no element of order 2. Since $W H_{4}=\sigma_{2}^{2}$ and $W H_{2}^{2}=0$, we conclude $l$ cannot be odd either.

(b) $H=\mathrm{SO}(2 l+1)$ or $\operatorname{Spin}(2 l+1)$.

$Z(\operatorname{Spin}(2 l+1)) \simeq Z / 2$ and $Z(\operatorname{SO}(2 l+1))=0$. Since calculations are done in the adjoint group, we need only consider the case of $\operatorname{SO}(2 l+1)$. Here we simply notice that $\mathrm{WH}_{2}=\sigma_{2}$ and $\mathrm{Ker} i^{*}$ contains no element of this degree. So $H=B_{l}$ does not occur.

(c) $H=\operatorname{Sp}(l)$.

$Z(\operatorname{Sp}(l)) \simeq Z / 2$ with generator $-I$, where $I$ is the identity matrix. Now $W H_{4}=(l+1) \sigma_{1}^{4}, W H_{2}^{2}=\dot{0}$, and $W H_{1}^{2}=0$. So if $l$ is even, $W H_{4}=$ $\sigma_{1}^{4}$, which is not 0 even after removing the center. Hence $l$ must be odd. In this case we compute 2-weights using the corank 12 -torus in the standard maximal 2-torus as explained before. This 2-torus does not contain the generator of the center. Here we have $\tilde{W} H_{8}=l \sigma_{2}^{4}$, while $\tilde{W} H_{4}^{2}=0$. So $l$ cannot be odd either.

(d) $H=\operatorname{SO}(2 l)$ or $\operatorname{Spin}(2 l)$.

Now $Z(\operatorname{Spin}(2 l))=Z / 4$ or $Z / 2+Z / 2$ and $Z(\operatorname{SO}(2 l))=Z / 2$. Again we perform our calculations on $\mathrm{SO}(2 l)$ since actually this should be performed on the adjoint group. $W H_{4}=(l+1) \sigma_{2}^{2}$ and $W H_{1}^{2}=0=W H_{2}^{2}$. So $l$ must be odd because $W H_{4} \neq 0$ even if we stay away from the generator of $Z(\mathrm{SO}(2 l))$. For $l$ odd, we examine $W H_{8}=\sigma_{4}^{2}+\sigma_{3} \sigma_{5}+C \sigma_{2}^{4}$, where $C=0,1 . W H_{1}=W H_{2}=W H_{3}=0=W H_{4}$. Therefore, $W H_{8} \neq 0$.

(e) $H=G_{2}$.

$Z\left(G_{2}\right)=0$. From $\left(W G_{2}\right)_{*}$ we see that $\left(W G_{2}\right)_{8} \neq 0$.

(f) $H=F_{4}, E_{6}, E_{7}, E_{8}$.

For these cases we avoid specific computation by noticing the following:

If $P\left(\sigma_{1}, \ldots, \sigma_{l}\right)$ is a homogeneous polynomial over a field $k$ in the elementary symmetric polynomials $\left\{\sigma_{i}\right\}$ in the variables $y_{1}, \ldots, y_{l}$, and $P=P_{1}+\cdots+P_{N}$ is the decomposition of $P$ into homogeneous polynomials of the same degree, then if, for all $i, P_{l} \equiv 0 \bmod P_{1}^{2}, \ldots, P_{[l / 2]}^{2}$, then actually $P_{t}=0$ for all $i$.

In view of this, we need only show that $(W H)_{*}$ does not equal 1 . We stress that by $P_{l}=0$ we mean $P_{i}$ is the zero polynomial and not a 
polynomial that evaluates to be 0 . We have shown $\left(W F_{4}\right)_{*} Z 1$. For $E_{6}$ we use the maximal 2-torus of $A_{1} \times A_{5}$ in $E_{6}$, which is contained in that of $E_{6}$. So the 2-weights of $\operatorname{Ad} E_{6} \mid A_{1} \times A_{5}=\operatorname{Ad~} A_{1}+\operatorname{Ad} A_{5}+\Lambda^{3} \mu_{6}$ are $\lambda$, $y_{i}+y_{j}$ with $i<j$ and multiplicity $2, y_{i}+y_{j}+y_{k}+\lambda$ with $i<j<k$ and multiplicity 2 . So

$$
W_{*}=(1+\lambda)^{2} \prod_{i<j}\left(1+y_{i}+y_{j}\right)^{2} \prod_{i<j<k}\left(1+y_{i}+y_{j}+y_{k}+\lambda\right)^{2} .
$$

Take $\left(\lambda, y_{1}, \ldots, y_{6}\right)=(0,1,1,0,0,0,0)$ and evaluate $W_{*}$ at this point. We get 0 ; on the other hand, $W_{*}=1+W_{1}+W_{2}+\cdots+W_{N}$. So some $W_{i}$ must be nonzero. Now $Z\left(E_{6}\right)=Z / 3$, so it does not contain elements of order 2.

For $E_{8}$ we take the maximal 2-torus of $A_{8} \subseteq E_{8}$. Since Ad $E_{8} \mid A_{8}=$ Ad $A_{8}+\Lambda^{3} \mu_{9}+\bar{\Lambda}^{3} \mu_{9}$, the 2-weights are $y_{i}+y_{j}$ with $i<j$ and $y_{i}+y_{j}+y_{k}$ with $i<j<k$, with multiplicity 2 . Hence

$$
W_{*}=1+W_{1}+W_{2}+\cdots=\prod_{i<j}\left(1+y_{i}+y_{j}\right)^{2} \prod_{i<j<k}\left(1+y_{i}+y_{j}+y_{k}\right)^{2} \text {. }
$$

We evaluate at $(1,1,0, \ldots, 0)$. Then $W_{*}=0$ and so some $W_{i}$ must be nonzero. Notice that $Z\left(E_{8}\right)=0$. The argument for $E_{7}$ proceeds analogously. We use the maximal 2-torus of $A_{7} \subseteq E_{7}$ and the fact that Ad $E_{7} \mid A_{7}=\operatorname{Ad} A_{7}+\Lambda^{4} \mu_{8}$. Although $Z\left(E_{7}\right)=Z / 2$, we have enough points to choose from for the evaluation argument.

2. Again we may use reduction 2 in [4]. It follows that we are reduced to case 1 , which cannot occur.

3. $\Psi$ is injective and $W H_{4}=l \sigma_{2}^{2}$, while the 2-weights of $\Psi$ are $y_{i}$ with multiplicity 2 . Hence $\left((W \Psi)_{2}^{2}, \ldots, W \Psi_{l}^{2}\right)=\left(\sigma_{2}^{4}, \ldots, \sigma_{l}^{4}\right)$ implies $W H_{4} \neq 0$ mod the ideal if $l$ is odd.

4. The 2-weights are $y_{1}, \ldots, y_{l}$ with multiplicity 1 and so $\left(W \Psi_{2}^{2}, \ldots, W \Psi_{l}^{2}\right)=\left(\sigma_{2}^{2}, \ldots, \sigma_{l}^{2}\right)$. For $l$ odd,$W H_{2}=\sigma_{2} \neq 0$.

5. The 2-weights are $y_{1}, \ldots, y_{l}$ with multiplicity 2. $\left(W \Psi_{2}^{2}, \ldots, W \Psi_{l}^{2}\right)$ $=\left(\sigma_{2}^{4}, \ldots, \sigma_{2}^{4}\right)$. Since $W H_{4}=(l+1) \sigma_{1}^{4}, l$ cannot be even.

6. Let $\Lambda_{2}$ denote the 7-dimensional representation of $G_{2}$. Then $W \Lambda_{2 *}=1+A+B+C$ (for notation see the proposition in \$2) and so one sees that $\left(W G_{2}\right)_{8}=\left(W 2 \Lambda_{2}\right)_{8} \neq 0 \bmod \left(\left(W 2 \Lambda_{2}\right)_{1}^{2}, \ldots,\left(W 2 \Lambda_{2}\right)_{4}^{2}\right)$.

7. Let $A, B, C$ and $A^{\prime}, B^{\prime}, C^{\prime}$ be, respectively, the expressions given in the proposition in $\$ 2$ for two copies of $G_{2}$. Then

$$
W H_{*}=\left(1+A^{2}+B^{2}+C^{2}\right)\left(1+A^{\prime 2}+B^{\prime 2}+C^{\prime 2}\right),
$$

and if $\Psi=2\left(\Lambda_{2}+\Lambda_{2}^{\prime}\right)$, then

$$
W \Psi_{*}^{2}=\left(1+A^{4}+B^{4}+C^{4}\right)\left(1+A^{\prime 4}+B^{\prime 4}+C^{\prime 4}\right),
$$

so clearly this case is impossible. 
8. If $H=\mathrm{SU}(3) \times \mathrm{SU}(3)$ and $\sigma_{i}$ and $\tau_{i}$ are, respectively, symmetric polynomials corresponding to each factor of $\mathrm{SU}(3)$. A straightforward computation shows that

$$
W H_{*}=1+\left(\sigma_{2}^{2}+\tau_{2}^{2}\right)+\left(\sigma_{3}^{2}+\tau_{3}^{2}\right)+\left(\sigma_{2}^{2} \tau_{2}^{2}\right)+\left(\sigma_{3}^{2} \tau_{3}^{2}+\sigma_{2}^{2} \tau_{3}^{2}\right)+\sigma_{3}^{2} \tau_{3}^{2} .
$$

If $\Psi$ is one of the representations listed, then $W \Psi_{*}^{2}=W H_{*}^{2}$ and we see that this case cannot occur.

9. This case is similar to (3).

10. $W H_{*}=1+\sigma_{2}^{2}+\sigma_{3}^{2}$. If $k+l=6$, the 2-weights are the same as those of $6 \mu_{3}$, which are $y_{1}, y_{2}, y_{3}$ with multiplicity 6 . Therefore,

$$
W \Psi_{*}^{2}=1+\sigma_{2}^{4}+\sigma_{3}^{4}+\sigma_{2}^{8}+\left(\sigma_{3}^{8}+\sigma_{2}^{12}\right)+\sigma_{2}^{4} \sigma_{3}^{8}+\sigma_{3}^{4} \sigma_{2}^{8}+\sigma_{3}^{12} .
$$

Thus the vanishing condition is not satisfied.

Proposition 2. Let $\mathrm{Sp}(m)$ act smoothly on a manifold such that the first three Pontrjagin classes and all Stiefel-Whitney classes vanish. Let $\Psi$ : $H \rightarrow \mathrm{Sp}(m)$ be an almost faithful symplectic representation of a compact connected Lie group $H$. Then $(H, \Psi)$ in the following cannot have $\Psi(H)$ as the connected principal isotropy type of the $\mathrm{Sp}(m)$-action:

1. $H=\mathrm{SU}(n), n=3,4,5, \Psi=\mu_{n}+\bar{\mu}_{n}$.

2. $H=\mathrm{SU}(3) \times \mathrm{SU}(3), \Psi=\mu_{3}+\bar{\mu}_{3}+\mu_{3}^{\prime}+\bar{\mu}_{3}^{\prime}$.

3. $H=G_{2} \times G_{2}, \Psi=2\left(\Lambda_{2}+\Lambda_{2}^{\prime}\right)$, where $\Lambda_{2}$ is the 7-dimensional representation of $G_{2}$.

4. $H=G_{2}, \Psi=2 \Lambda_{2}$.

Proof. The calculations are exactly the same as those for the previous proposition.

Proposition 3. Hypotheses as in Proposition 2 except with $\mathrm{Sp}(m)$ replaced by $\mathrm{SO}(m)$. Then the following pairs $(H, \Psi)$ cannot have $\Psi(H)$ as the connected principal isotropy type of the $\mathrm{SO}(m)$-action:

1. $H=G_{2}, \Psi=4 \Lambda_{2}$.

2. $H=\mathrm{SU}(3), \mathrm{SU}(5), \Psi=2\left(\mu_{n}+\bar{\mu}_{n}\right)$.

3. $H=\mathrm{SU}(3) \times \mathrm{SU}(3), \Psi=2\left(\mu_{3}+\mu_{3}^{\prime}+\bar{\mu}_{3}+\bar{\mu}_{3}^{\prime}\right)$.

4. $H=\mathrm{Sp}(n), n$ even, $\Psi=2 v_{n}$.

5. $H=\mathrm{SO}(n), n$ odd,$\Psi=2 \rho_{n}$.

6. $H$ is semisimple and contains simple normal factors of type $B_{n}, C_{2 n}$, $D_{2 n}, \Psi=2 \operatorname{Ad} \Psi(H)$.

Proof. Again the calculations are similar to those of Proposition 1 and so are omitted. 
As an application of these propositions, let us prove

Proposition 4. Let $G=\mathrm{Sp}(m)$ act smoothly on $W_{n, n-k}=$ complex Stiefel manifold of orthonormal $n$ - $k$ planes in $C^{n}$ with $k \geq n / 2, n \geq 11$, and $\operatorname{dim} \mathrm{Sp}(m) \geq \operatorname{dim} \mathrm{SU}(n)$. Then the action is trivial.

Proof. Combining Proposition 2 in this section with Theorem 2 in [4] we conclude that the connected principal isotropy type of the action must be one of the following:

1. any torus $T$,

2. $\nu_{l}(\mathrm{Sp}(l))$,

3. $r\left(\nu_{1}^{(1)}+\cdots+\nu_{1}^{(l)}\right)\left(\operatorname{Sp}(1)^{(1)} \times \cdots \times \operatorname{Sp}(1)^{(l)}\right), r=1,2,4$. In case 1 , if $\left(H^{0}\right)$ is the connected principal isotropy type, then $\operatorname{dim} H^{0} \leq m$. One easily sees that $m \leq \sqrt{3 / 8} n<0.615 n$. In case $3, \operatorname{dim} H^{0} \leq 3 r l \leq 3 m$ and one checks that $m<0.615 n+1$. On the other hand, $n \geq 11$ and $k \geq n / 2$ together with $\operatorname{dim} \operatorname{Sp}(m) \geq \operatorname{dim} \mathrm{SU}(n)$ imply $m \geq 0.615 n+1$. Hence case 2 must occur and, by Theorem $\mathrm{Cl}$ of $\$ 8$ of [4], all connected isotropy groups are of the type $\nu_{l_{x}}\left(\mathrm{Sp}\left(l_{x}\right)\right), l_{x} \geq l$, where $l$ is the rank of the connected principal isotropy type. If $G(x)$ is any orbit, then, since it is covered by a quaternionic Stiefel manifold, $\pi_{i}(G(x))=0$ whenever $i \leq 4 l$ +2 . Consequently, $\tilde{H}(G(x) ; Q)=0$ for $i \leq 4 l+2$. By the Vietoris mapping theorem (see for example, $\S 6.4$, p. 142 of [3]) we have

$$
H^{i}\left(W_{n, n-k} / G ; Q\right) \simeq{p^{*}}^{l} H^{l}\left(W_{n, n-k} ; Q\right) \text { if } i \leq 4 l+2,
$$

where $p$ is the orbit map. Therefore, there are nonzero elements $a_{k}, \ldots, a_{q}$ in $H^{2 l+1}\left(W_{n, n-k} / G ; Q\right), q=\min (2 l, n-1), \operatorname{deg} a_{l}=2 i+1$, so that $p^{*} a_{i}=x_{i}$ in $H^{2 i+1}\left(W_{n, n-k} ; Q\right)$, where $H^{*}\left(W_{n, n-k} ; Q\right) \simeq \Lambda\left(x_{k}, \ldots, x_{n-1}\right)$. Hence $a_{k} \cdots a_{q} \neq 0$. But note that

$$
\begin{aligned}
\operatorname{dim}\left(W_{n, n-k} / G\right) & =n^{2}-k^{2}-\left(2 m^{2}+m-2 l^{2}-l\right) \\
& \leq\left(4 l^{2}-k^{2}\right)+\left(-2 l^{2}+l+1\right) .
\end{aligned}
$$

If $l>1,-2 l^{2}+l+1<0$, so

$$
\operatorname{dim}\left(W_{n, n-k} / G\right)<4 l^{2}-k^{2}<\operatorname{dim}\left(a_{k} \cdots a_{q}\right) \quad \text { if } 2 l=q,
$$

or equivalently, $n-1 \geq 2 l$, which is a contradiction. So $n-1<2 l$, in which case $\operatorname{dim}\left(W_{n, n-k} / G\right)=n^{2}-k^{2}$, implying $l=m$, or, equivalently, the action is trivial. 
Added in Proof. This paper was written in 1979 before the author's thesis. Circumstances have delayed its publication. Meanwhile actions on the complex Stiefel manifolds $W_{n, 2}, n$ odd, were studied more extensively in the author's papers listed below.

[6] Trans. Amer. Math. Soc., 272 (1982), 589-610, 611-628.

[7] Canad. Math. Soc. Conf. Proc., Vol. 2, Part 2, (1982), 303-311.

\section{REFERENCES}

[1] A. Borel and F. Hirzebruch, Characteristic Classes and Homogeneous Spaces I, Amer. J. Math., 80 (1958), 458-538; II, ibid. 81 (1959), 315-382; III, ibid. 82 (1960), 491-504.

[2] - Sous-groupes commutatifs et torsion des groupes de Lie compacts connexes, Tohoku Math. J., 13 (1961), 216-240.

[3] G. Bredon, Sheaf Theory, McGraw Hill (1967).

[4] Wu-Yi Hsiang, On Characteristic Classes of Compact Homogeneous Spaces and Their Application in Compact Transformation Groups I, Bol. Soc. Brasil. Mat., 10 (1979), 87-161.

[5] _ Cohomology Theory of Topological Transformation Groups, Ergebnisse der Mathematik und ihrer Grenzgebiete, Band 85, Springer-Verlag (1970).

Received October 16, 1981.

DePartment OF Mathematical SCIENCES

MCMASTER UNIVERSITY

Hamilton, ONTARIO, Canada L8S 4K1 



\title{
PACIFIC JOURNAL OF MATHEMATICS EDITORS
}

\author{
Donald BabBITT (Managing Editor) \\ University of California \\ Los Angeles, CA 90024 \\ Hugo Rossi \\ University of Utah \\ Salt Lake City, UT 84112 \\ C. C. Moore and Arthur Ogus \\ University of California \\ Berkeley, CA 94720
}

\author{
J. Dugundu \\ Department of Mathematics \\ University of Southern California \\ Los Angeles, CA 90089-1113
}

R. FINN and H. SAMELSON

Stanford University

Stanford, CA 94305

\section{ASSOCIATE EDITORS}
R. ARENS
E. F. BECKENBACH
B. H. NeumanN
F. WolF
K. YosHIDA (1906-1982)

\section{SUPPORTING INSTITUTIONS}

\author{
UNIVERSITY OF ARIZONA \\ UNIVERSITY OF BRITISH COLUMBIA \\ CALIFORNIA INSTITUTE OF TECHNOLOGY \\ UNIVERSITY OF CALIFORNIA \\ MONTANA STATE UNIVERSITY \\ UNIVERSITY OF NEVADA, RENO \\ NEW MEXICO STATE UNIVERSITY \\ OREGON STATE UNIVERSITY
}

\author{
UNIVERSITY OF OREGON \\ UNIVERSITY OF SOUTHERN CALIFORNIA \\ STANFORD UNIVERSITY \\ UNIVERSITY OF HAWAII \\ UNIVERSITY OF TOKYO \\ UNIVERSITY OF UTAH \\ WASHINGTON STATE UNIVERSITY \\ UNIVERSITY OF WASHINGTON
}

The Supporting Institutions listed above contribute to the cost of publication of this Journal, but they are not owners or publishers and have no responsibility for its content or policies.

Mathematical papers intended for publication in the Pacific Journal of Mathematics should be in typed form or offset-reproduced (not dittoed), double spaced with large margins. Please do not use built up fractions in the text of the manuscript. However, you may use them in the displayed equations. Underline Greek letters in red, German in green, and script in blue. The first paragraph must be capable of being used separately as a synopsis of the entire paper. In particular it should contain no bibliographic references. Please propose a heading for the odd numbered pages of less than 35 characters. Manuscripts, in triplicate, may be sent to any one of the editors. Please classify according to the scheme of Math. Reviews, Index to Vol. 39. Supply name and address of author to whom proofs should be sent. All other communications should be addressed to the managing editor, or Elaine Barth, University of California, Los Angeles, California 90024.

There are page-charges associated with articles appearing in the Pacific Journal of Mathematics. These charges are expected to be paid by the author's University, Government Agency or Company. If the author or authors do not have access to such Institutional support these charges are waived. Single authors will receive 50 free reprints; joint authors will receive a total of 100 free reprints. Additional copies may be obtained at cost in multiples of 50 .

The Pacific Journal of Mathematics is issued monthly as of January 1966. Regular subscription rate: $\$ 132.00$ a year (6 Vol., 12 issues). Special rate: $\$ 66.00$ a year to individual members of supporting institutions.

Subscriptions, orders for numbers issued in the last three calendar years, and changes of address should be sent to Pacific Journal of Mathematics, P.O. Box 969, Carmel Valley, CA 93924, U.S.A. Old back numbers obtainable from Kraus Periodicals Co., Route 100, Millwood, NY 10546.

The Pacific Journal of Mathematics ISSN 0030-8730 is published monthly by the Pacific Journal of Mathematics at P.O. Box 969, Carmel Valley, CA 93924. Application to mail at Second-class postage rates is pending at Carmel Valley, California, and additional mailing offices. Postmaster: Send address changes to Pacific Journal of Mathematics, P. O. Box 969, Carmel Valley, CA 93924.

PUBLISHED BY PACIFIC JOURNAL OF MATHEMATICS. A NON-PROFIT CORPORATION

Copyright $\odot 1984$ by Pacific Journal of Mathematics 


\section{Pacific Journal of Mathematics}

\section{Vol. 112, No. $2 \quad$ February, 1984}

Kenneth F. Andersen and Wo-Sang Young, On the reverse weak type inequality for the Hardy maximal function and the weighted classes

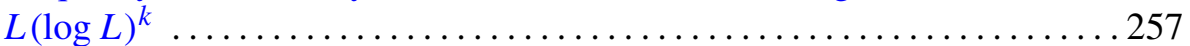

Richard Eugene Bedient, Double branched covers and pretzel knots ..... 265

Harold Philip Boas, Holomorphic reproducing kernels in Reinhardt

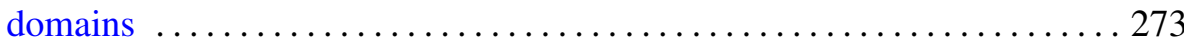

Janey Antonio Daccach and Arthur Gabriel Wasserman, Stiefel's theorem and toral actions ................................. 293

Michael Fried, The nonregular analogue of Tchebotarev's theorem ....... 303

Stanley Joseph Gurak, Minimal polynomials for circular numbers . . . . . . 313

Norimichi Hirano and Wataru Takahashi, Nonlinear ergodic theorems for an amenable semigroup of nonexpansive mappings in a Banach space . . 333

Jim Hoste, Sewn-up $r$-link exteriors . . . . . . . . . . . . . . . . . . . 347

Mohammad Ahmad Khan, The existence of totally dense subgroups in

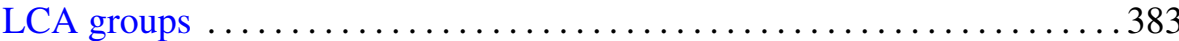

Mieczysław Kula, Murray Angus Marshall and Andrzej Sładek, Direct

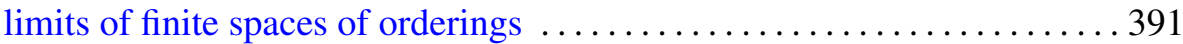

Luis Montejano Peimbert, Flat Hilbert cube manifold pairs ........... 407

Steven C. Pinault, An a priori estimate in the calculus of variations . . . . . 427

McKenzie Y. K. Wang, Some remarks on the calculation of Stiefel-Whitney classes and a paper of Wu-Yi Hsiang's

Brian Donald Wick, The calculation of an invariant for Tor . . 445

Wolfgang Wollny, Contributions to Hilbert's eighteenth problem 